\title{
A HYPOTHESIS ON THE RICHNESS OF A GALAXY IN BINARY STELLAR SYSTEMS
}

\author{
Hans Zinnecker
}

Max-Planck-Institut für Physik und Astrophysik Institut für Extraterrestrische Physik D-8046 Garching, W. -Germany

\section{INDRODUCTION}

This contribution is concerned with the origin of binary stellar systems (cf. Abt 1977, Huang 1977). The hierarchical fragmentation scheme of rotating interstellar clouds, proposed by Bodenheimer (1978), is combined with the role of magnetic fields in the early stages of star formation (Mouschovias 1978, Dorfi 1981). The possible influence of the local strength of the mean interstellar magnetic field on the local fraction of binary systems in a galaxy is stressed.

\section{THE BASIC IDEA}

The hierarchical scheme of Bodenheimer (1978) is conceived to solve the angular momentum problem of star formation in several steps by conversion of the spin angular momentum of a fragment into orbital angular momentum of two or more subfragments, without transport of angular momentum. It is based on successive 'ring formation and ring fragmentation' during hydrodynamic collapse (Larson 1972, Tohline 1980, Norman and Wilson 1978). The effect of magnetic fields is not incorporated in the scheme. Magnetic fields essentially cause a magnetic braking of the rotation of diffuse interstellar clouds up to a gas density at which ambipolar diffusion (Mestel and Spitzer 1956) starts to decouple the magnetic field from the bulk of the gaseous matter (e.g. Meste1 1977, Spitzer 1978). This is believed to take place at a gas density of about $10^{-19} \mathrm{~g} / \mathrm{cm}^{3}$, where the fractional degree of ionization becomes low enough, i.e. of order $10^{-8}$ (cf. Nakano 1979, Elmegreen 1979). Up to this density the torques of the frozen-in magnetic field help to solve the angular momentum problem of star formation (Mestel and Paris 1979, Mouschovias and Paleologou 1980). After the decoupling the residual angular momentum problem may be solved along the lines of hierarchical fragmentation suggested by Bodenheimer (1978) which is supported by the recent observations of Fekel (1981).

It is the basic idea of this note to point out the hypothesis that the fraction of binary systems in a galaxy may depend on how much of the angular momentum problem of star formation can be solved by magnetic 
torques, and how much of the angular momentum problem is left over to the cascade process of Bodenheimer (1978). Of course, the issue that I have raised should actually be discussed in terms of mechanisms promoting binary star formation versus mechanisms promoting single star formation. However, as long as astrophysicists do not have a theory of turbulence and angular momentum transport, it is very difficult to assess the importance of the mechanisms promoting single star formation (cf. Tscharnuter 1980). Here I am dealing only with the first half of the problem; the conclusion will be tentative to the extent that I sha11 neglect the second half of the problem.

The amount of magnetic braking depends on the critical gas density $P_{\text {crit }}$
above which magnetic braking becomes inefficient due to the ambipolar diffusion of the magnetic field; Pcrit is determined by the requirement that the braking timescale is equal to the ambipolar diffusion timescale. According to equ. (12) in Mouschovias (1978) this requirement leads to

$$
\rho_{\text {crit }} \propto M_{c 1} n_{\text {ion }}^{3 / 2} \bar{B}^{3 / 2}
$$

where $\mathrm{M}_{\mathrm{Cl}}$ is the cloud mass, $\mathrm{n}_{\text {ion }}$ is the number density of charged particles, and $\bar{B}$ is the mean interstellar magnetic field. From the above formula one may infer a systematic tendency that the higher the mean interstellar magnetic field the more magnetic braking occurs (on the implicit assumption that the other parameters remain constant). For this reason it is interesting theoretically to know the local strength of the mean interstellar magnetic field. The following model of the local strength of the mean interstellar magnetic field is presented: Imagine a uniform primordial seed magnetic field which gets amplified during the nonhomologous collapse of the proto-galaxy in a magneto-hydrokinematic manner (the energy density in the galactic magnetic field is always much less than the gravitational energy density of the galaxy). From conservation of mass and magnetic flux it is found (e.g. Mestel $1965)$ that the local mean magnetic field strength $\bar{B}(r)$ is proportional to the $2 / 3$ th power of the local mean interstellar gas density $\bar{\rho}(r)$ in a galaxy, i.e.

$$
\bar{B}(r) \propto[\bar{\rho}(r)]^{2 / 3} \text {. }
$$

$r$ denotes the galacto-centric distance (either in a spiral or in an elliptical galaxy). Equs. (1) and (2) combine to the simple relation

$$
\rho_{\text {crit }} \propto \bar{p}(r)
$$

which says how the local effect of the magnetic field in star formation is related to the global density structure of a galaxy (the gas density in a galaxy is roughly proportional to the total matter density).

\section{PREDICTION}

From the foregoing considerations I predict that there is a gradient of the percentage of binary stellar systems in a galaxy, in the sense that 
the central regions will not be as rich in binary systems as the outer regions. It might be that the dark halos of galaxies consist of a very large number of very low-mass binary systems (cf. Zinnecker $1981 \mathrm{a}, \mathrm{b}$ ).

\section{REFERENCES}

Abt H. (1977): Revista Mexicana de Astronomia y Astrofisica Vol.3

(IAU-Colloqu. No. 33 Special Issue)

Bodenheimer P. (1978): Ap. J. 224, 488.

Dorfi E. (1981): Ph.D. Thesis (Univ. Wien)

Elmegreen B.G. (1979): Ap. J. 232, 729.

Feke1 F. (1981): Ap.J. 246, $87 \overline{9}$.

Huang S.S. (1977): Revista Mexicana de Astronomia y Astrofisica Vo1. 3

(IAU-Co1loqu. No. 33 Special Issue)

Larson R.B. (1972): M.N.R.A.S. 156, 437.

Mestel L. (1965): Quart. J. Roy. Astr. Soc. 6, 265.

Meste1 L. (1977): IAU-Symp. No. 75 (de Jong $\overrightarrow{\mathrm{T}}$. and Maeder A., eds.), p. 213 .

Mestel L. and Spitzer L. (1956): M.N.R.A.S. 116, 503 . Mestel L. and Paris R.B. (1979): M.N.R.A.S. $\overline{187}, 337$.

Mouschovias T. (1978): in Protostar and Planets (Gehrels T., ed.),p.209. Mouschovias T. and Paleologou (1980): The Moon and the Planets 22, 31 . Nakano T. (1979): P.A.S.J. 31, 697.

Norman M.L. and Wilson J.R. (1978): Ap. J. 224, 497.

Spitzer L. (1978): Physica1 Processes in the Interstellar Medium (Wiley, New York)

Tohline J.E. (1980): Ap. J. 236, 160.

Tscharnuter W.M. (1980): IAU-Symp. No. 93 (Kyoto).

Zinnecker H. (1981a): Ph.D. Thesis (TU München)

Zinnecker $\mathrm{H}$. (1981b): in preparation 